\title{
Somatosensory and brainstem auditory evoked potentials in cardiac arrest patients treated with hypothermia*
}

Marjaana Tiainen, MD; Tero T. Kovala, MD, PhD; Olli S. Takkunen, MD, PhD; Risto 0. Roine, MD, PhD

Objective: To evaluate the prognostic value of short-latency median nerve somatosensory evoked potentials and brainstem auditory evoked potentials in outcome prediction for comatose cardiac arrest patients treated with hypothermia.

Design: Prospective, randomized, controlled trial of mild hypothermia after out-of-hospital cardiac arrest; a substudy of the European Hypothermia After Cardiac Arrest study.

Setting: Intensive care unit of a tertiary referral hospital (Helsinki University Central Hospital).

Patients: Sixty consecutive patients (aged 18-75 yrs) resuscitated from out-of-hospital ventricular fibrillation and comatose at $\mathbf{2 4} \mathrm{hrs}$ after cardiac arrest; all patients were randomly assigned either to therapeutic hypothermia of $33^{\circ} \mathrm{C}$ or normothermia.

Interventions: All patients received standard intensive care for at least 2 days. Patients randomized to hypothermia were cooled with an external cooling device for $24 \mathrm{hrs}$ and then allowed to rewarm slowly for 12 hrs. In the normothermia group, the core temperature was kept below $38^{\circ} \mathrm{C}$ with antipyretics and by phys- ical means. The clinical outcome was assessed 6 months after cardiac arrest.

Measurements and Main Results: Somatosensory evoked potentials and brainstem auditory evoked potentials were recorded 24-28 hrs after cardiac arrest. All wave latencies were significantly prolonged in the hypothermia group. Bilaterally absent N20 waves predicted permanent coma with a specificity of $100 \%$ in both treatment groups. Brainstem auditory evoked potential recordings did not correlate with the outcome in either treatment group.

Conclusions: The prognostic ability of median nerve shortlatency somatosensory evoked potentials does not seem to be affected by therapeutic hypothermia. Brainstem auditory evoked potentials had no additional value in outcome prediction. (Crit Care Med 2005; 33:1736-1740)

KEY WoRDS: cardiac arrest; hypothermia; somatosensory evoked potentials; brainstem auditory evoked potentials; hypoxic-ischemic encephalopathy; prognosis
A dvances in emergency medicine have increased the number of cardiac arrest patients in the emergency department. The duration and severity of global cerebral ischemia and secondary mechanisms of ischemia related to reperfusion contribute to the extent of brain damage. Mild hypothermia of $33^{\circ} \mathrm{C}$ for 12 or $24 \mathrm{hrs}$ has been shown to improve neurologic outcome after cardiac arrest $(1,2)$ and also to increase the chance of survival (1).

*See also p. 1868.

From the Department of Neurology, Helsinki University Central Hospital (MT, ROR); EMG Laboratories, Ltd. (TTK); and Department of Anesthesiology and Intensive Care Medicine, Helsinki University Central Hospital and Kuopio University Hospital (OST), Helsinki, Finland.

None of the authors has anything to disclose. Supported in part by the Finnish Neurology Foundation, the Clinical Research Institute of Helsinki University Central Hospital, Maire Taponen Foundation, Laerdal Foundation, and Helsinki University Central Hospital, Finland. Kinetic Concepts, Inc., Wareham, UK, provided the cooling device used in the study.

Copyright (C) 2005 by the Society of Critical Care Medicine and Lippincott Williams \& Wilkins

DOI: 10.1097/01.CCM.0000171536.63641.D9
Early prediction of neurologic outcome for patients resuscitated from cardiac arrest is a challenging task. The median nerve somatosensory evoked potentials (SEPs) have been reported to accurately predict poor outcome after cardiac arrest (3, 4). Hypothermia is known to prolong all neuronal conduction velocities, including the SEP and brainstem auditory evoked potential (BAEP) latencies, and temperature has also been reported to affect the amplitudes of SEP responses (5-8). Mild to moderate hypothermia does not abolish cortical responses to sensory evoked potentials (9). The cortically generated component N20 has been shown to disappear only at mean nasopharyngeal temperature of $20^{\circ} \mathrm{C}$ under normal hemodynamic conditions $(6,10)$. The measurement of BAEPs has been used to monitor neuronal function at the level of the brainstem. Hypothermia increases the BAEP interwave latencies $(5,11,12)$. BAEP components have also been reported to be abolished at temperatures below $20^{\circ} \mathrm{C}(12)$.

The aim of this study was to evaluate the prognostic value of the short-latency median nerve SEPs and BAEPs in out- come prediction for comatose cardiac arrest patients treated with hypothermia.

\section{SUBJECTS AND METHODS}

The protocol and consent procedure of this study was approved by the ethics committee of Helsinki University Central Hospital in accordance with institutional guidelines. A deferred consent was used for all patients. The patient's family was informed about the trial and could withdraw the patient at any time from the study. Each patient was informed about the trial both orally and in writing, when he or she was able to receive this information.

Patients randomized into the Hypothermia After Cardiac Arrest trial were included (1). All adult patients admitted to the emergency department of Helsinki University Central Hospital after resuscitation from out-of-hospital cardiac arrest were screened for the trial. The criteria for inclusion were an age of 18-75 yrs, a witnessed cardiac arrest, ventricular fibrillation or nonperfusing tachycardia as the initial cardiac rhythm, presumed cardiac origin of the arrest, an estimated interval of 5-15 mins from collapse to the first attempt at resuscitation by emergency medical personnel, and an interval of $<60$ mins from collapse to restoration of spontaneous circulation (ROSC). If 
cardiac arrest occurred after the arrival of the first responding unit or the patient responded to verbal command after ROSC, the patient was excluded. Additional exclusion criteria were cardiac arrest due to intoxication or trauma; tympanic temperature $<30^{\circ} \mathrm{C}$ at admission; evidence of hypotension (mean arterial pressure $<60 \mathrm{~mm} \mathrm{Hg}$ ) for $>30$ mins after ROSC and before randomization; evidence of hypoxia (arterial oxygen saturation $<85 \%$ ) for $>15$ mins after ROSC and before randomization; a preceding terminal illness; known preexisting coagulopathy; pregnancy; factors that made participation in follow-up unlikely; and enrollment in another study (1).

All cardiac arrest data were collected in the Utstein style (13). Cardiac arrest was defined as the absence of both palpable pulse and spontaneous respiration. ROSC was defined as return of palpable arterial pulse. Within the Helsinki metropolitan area, basic life support and advanced cardiac life support were provided by the three-tiered Helsinki emergency medical services. Outside the city of Helsinki, basic life support was provided by the staff of regional fire brigade-based emergency medical services and advanced cardiac life support by the emergency medical helicopter. All emergency medical service units are equipped with semiautomatic defibrillators, and the staffs are trained to intubate, insert intravenous catheters, and start medication.

Patients were transferred to the intensive care unit (ICU) after assessment of respiratory and hemodynamic function and initial neurologic evaluation in the emergency department. All patients underwent standard intensive care management and monitoring, including mechanical ventilation and placement of arterial catheters, central venous catheters, and Foley catheters, with a temperature sensor and a pulmonary artery catheter used as necessary.

Sedation and analgesia were accomplished with administration of midazolam $(0.125$ $\mathrm{mg} / \mathrm{kg}$ per hr) and fentanyl $(0.002 \mathrm{mg} / \mathrm{kg} \mathrm{hr}$ intravenously), titrated for $32 \mathrm{hrs}$. To avoid shivering, pancuronium $(0.05 \mathrm{mg} / \mathrm{kg}$ per $\mathrm{hr})$ was initially used. The lowest dose that would permit complete muscle relaxation was used for a total of $32 \mathrm{hrs}$. Mean arterial pressure was targeted at $80 \mathrm{~mm} \mathrm{Hg}$. Decreases in systemic blood pressure were treated primarily with crystalloid fluids or hydroxyl ethyl starch. Inotropic agents were used if sufficient blood pressure control could not be achieved by fluid therapy alone. No glucose-containing solutions were infused. Intravenous insulin infusion was administered if blood glucose exceeded $10 \mathrm{mmol} / \mathrm{L}$, and normoglycemia was targeted. Optimal head-up position (30 degrees) was maintained.

Patients randomized to normothermia were allowed to rewarm passively to normo- thermia (core temperature, $<38^{\circ} \mathrm{C}$ ) and were then kept normothermic. Those randomized to hypothermia treatment were actively cooled externally to a core target temperature of $33^{\circ} \mathrm{C}$ (range, $32-34^{\circ} \mathrm{C}$ ) with a cooling device (Therakool, Kinetic Concepts, Wareham, UK). The cooling device consists of a mattress and cover that delivers cold air over the entire body. Ice packs were applied on the patient's axilla and groin to enhance the cooling. Hypothermia $\left(33^{\circ} \mathrm{C} \pm 1^{\circ} \mathrm{C}\right)$ was maintained for $24 \mathrm{hrs}$ from start of cooling, and patients were then allowed to rewarm for $12 \mathrm{hrs}$. Life support was maintained for at least 3 days for all patients and at least 7 days for patients responding to pain in any manner. The decision to withdraw treatment was based on clinical status. Withdrawal of care meant withdrawal of inotropic intravenous medications but maintenance of airway and ventilator treatment. The SEP and BAEP results were not available during the acute phase and thus did not affect the decision to withdraw treatment. Family members were informed of the decision to withdraw treatment but did not participate in the decision-making process.

Assessment of Outcome. Neurologic examination was performed daily during treatment in the ICU; on days 3,7 , and 14; at discharge from hospital; at 3 months; and at 6 months after cardiac arrest. The primary end point was the recovery of consciousness, defined as ability to obey verbal command, and the secondary outcome was favorable outcome at 6 months after cardiac arrest, as assessed by the Pittsburgh Outcome Scale $(14,15)$. This is a five-category scale of cerebral performance categories (CPCs). These categories are defined as follows: CPC 1, conscious and alert with normal cerebral function; CPC 2, conscious and alert with moderate cerebral function; CPC 3, conscious with severe cerebral disability; CPC 4, comatose or in persistent vegetative state; CPC 5 , dead. For statistical analyses, the neurologic outcome was dichotomized as good (CPC 1 and 2) or poor (CPC 3, 4 , and 5).

SEPS and BAEPs. The SEPs and BAEPs were recorded 24-28 hrs after cardiac arrest with a four-channel electromyography/evoked potentials device (Medelec Sapphire, Medelec Limited, Surrey, UK). For SEPs, square-wave pulses with duration of $0.2 \mathrm{msec}$ at a repetition rate of 3 pulses per sec were used as stimuli to the median nerve at wrist. Stimulus intensity was 25-30 mA. Ag/AgCl surface electrodes were placed according to the International 10-20 System as follows: for N9 peak on ipsilateral Erb's point (referenced to contralateral Erb's point), for N13 peak on cervical spinous process $\mathrm{C} 7$ (referenced to sternum), for P15 and N18 peaks on Fpz (referenced to ipsilateral mastoideum), and for N20 peak on contralateral parietal (referenced to ipsilateral parietal). A sweep time of $100 \mathrm{msec}$, an amplifier sensitivity of $20-50 \mu \mathrm{V} / \mathrm{div}$, a display sen- sitivity of $1.0-2.5 \mu \mathrm{V} / \mathrm{div}$, and a bandwidth of $20 \mathrm{~Hz}$ to $1 \mathrm{kHz}$ were used. Electrode impedances were maintained below $5 \mathrm{kOhm}$. Trials with excessive artefacts were rejected automatically. A mean 610 trials (range, 3501050) were averaged. Recordings were performed twice to both right and left median nerve stimulation. The distance between stimulation site and Erb's point was measured to calculate the nerve conduction velocity.

BAEPs were recorded with clicks of $105-\mathrm{dB}$ intensity presented monaurally at a rate of $9 / \mathrm{sec}$ through earphones. A mask of $20-\mathrm{dB}$ intensity was used in the contralateral ear. A sweep time of $10 \mathrm{msec}$, an amplifier sensitivity of $5-10 \mu \mathrm{V} / \mathrm{div}$, a display sensitivity of $0.05-$ $0.1 \mu \mathrm{V} / \mathrm{div}$, and a bandwidth of $100 \mathrm{~Hz}$ to 2 $\mathrm{kHz}$ were used. The $\mathrm{Ag} / \mathrm{AgCl}$ surface electrodes were placed on ipsilateral mastoideum and referenced to $\mathrm{Fz}$. Responses of 2048 stimuli were averaged for an individual test, and the procedure was performed twice to both right and left ear stimulation. The SEP and BEAP recordings were all analyzed retrospectively as a block. The physician analyzing SEPs and BAEPs was blinded to the treatment and outcome for the patient.

Statistical Analysis. Categorical variables are given as counts and percentages. Data are given as median and interquartile range. Means of left-sided and right-sided evoked potential stimulations were used for calculations. Amplitudes and latencies were calculated from cases with present responses. Continuous data were compared with the Mann-Whitney U-test. Outcome data are binary, and the chi-square test was used to compare proportions between the hypothermia and normothermia groups. $p<.05$ were considered statistically significant. We used the Statistica data analysis software system (StatSoft, Tulsa, OK) to analyze the data.

\section{RESULTS}

Seventy consecutive unconscious (Glasgow Coma Scale <9) patients met the inclusion criteria and were randomly assigned to hypothermia ( $\mathrm{n}=36$ ) or normothermia $(\mathrm{n}=34)$ treatment. In both groups one patient died before $24 \mathrm{hrs}$. SEPs to median nerve stimulation and BAEPs were recorded for 60 consecutive patients (subjects 9-70, with the exception of two patients who died before 24 hrs) at 24-28 hrs after cardiac arrest. SEP and BAEP recordings were not available for the first eight randomized patients for technical reasons, after which all consecutive patients surviving for at least 24 hrs were studied. Thirty of the 60 patients were randomized to the hypothermia treatment and 30 to the normothermia treatment. All patients were sedated, relaxated, and artificially ventilated during the evoked potentials recording 
session. The median core temperature during recording was $33.0^{\circ} \mathrm{C}$ in the hypothermia group and $37.5^{\circ} \mathrm{C}$ in the normothermia group.

Clinical and demographic data of patients $(\mathrm{n}=60)$ are presented in Table 1. No statistically significant differences were observed in any baseline characteristics between the two treatment groups. Forty-five patients recovered consciousness (26 in the hypothermia group and 19 in the normothermia group; $p=.04)$. At 6 months after cardiac arrest, the outcome was favorable for $77 \%(n=23)$ of the hypothermia-treated patients and $50 \%(\mathrm{n}=15)$ of the normothermiatreated patients $(p=.03)$.

SEP. Data from 57 patients (30 in the hypothermia group and 27 in the normothermia group) could be analyzed. For two normothermic subjects, reliable analysis of SEP recordings was prevented by excess muscle artefact due to insufficient relaxation, and one normothermic

Table 1. Clinical and demographic characteristics of patients treated with hypothermia or normothermia

\begin{tabular}{lcccc}
\hline & \multicolumn{3}{c}{ Median Value (Range) } \\
\cline { 2 - 5 } \multicolumn{1}{c}{ Characteristic } & $\begin{array}{c}\text { Hypothermia } \\
(\mathrm{n}=30)\end{array}$ & $\begin{array}{c}\text { Normothermia } \\
(\mathrm{n}=30)\end{array}$ \\
\hline Age, yrs & 60 & $(23-75)$ & 58 & $(18-75)$ \\
Male/female, no. (\%) & $26 / 4$ & $(87 / 13)$ & $22 / 8$ & $(73 / 27)$ \\
Bystander-initiated CPR, no. (\%) & 16 & $(53)$ & 16 & $(53)$ \\
BLS, min & 7 & $(4-14)$ & 7 & $(4-11)$ \\
ACLS, min & $14.5(7-59)$ & 13 & $(5-39)$ \\
ROSC, min & 17 & $(9-34)$ & $18.5(8-45)$ \\
Defibrillations before ROSC & 3 & $(1-12)$ & 2 & $(1-30)$ \\
Total dose of epinephrine before ROSC, mg & $2.0(0-9.0)$ & $2.0(0-6.5)$ \\
Glasgow Coma Scale score on admission & 5 & $(3-7)$ & 5 & $(3-8)$ \\
Blood glucose on admission, mmol/L & $10.0(6.1-18.7)$ & $9.7(4.9-25.9)$ \\
Tympanic temperature on admission, ${ }^{\circ} \mathrm{C}$ & $35.2(33.4-36.9)$ & $35.5(33.6-36.9)$ \\
\hline
\end{tabular}

CPR, cardiopulmonary resuscitation; BLS, basic life support; ACLS, advanced cardiac life support; ROSC, restoration of spontaneous circulation. patient had severe edema of the head and upper trunk, which made the analysis of SEP recordings impossible. The nerve conduction velocities, latencies to N13 responses, latencies to cortical N20 responses, and amplitudes of $\mathrm{N} 20$ responses and cervicocortical conduction times (times from cervicomedullary N13 to cortical N20) are presented in the Table 2. Nerve conduction velocity was statistically significantly lower in the hypothermia group $(p<.001)$, as expected. The latencies to N13 response and to early cortical N20 response were significantly longer in the hypothermia group $(p<$ $.001)$. The amplitudes of $\mathrm{N} 20$ responses were comparable in the two treatment groups and did not correlate with outcome. Cervical N13 peak was detected in all patients. The cortical N20 responses were bilaterally absent in 11 patients, of which three were in the hypothermia group and eight in the normothermia group. None of these patients regained (II, or Vwe At lenst one veforms I, III, or V were constantly missing in two hypothermia-treated and four normothermia-treated patients. In two patients these responses were missing only unilaterally; both patients were known to have a hearing loss and awakened later. In four patients these responses were missing bilaterally, and three of them did not regain consciousness. Of them, one patient had a hearing loss, which probably explained the findings in BAEP, since he had cortical N20 responses in SEPs. One patient also had bilaterally absent $\mathrm{N} 20$ responses on SEPs. One patient had severe edema of the head and upper trunk, and analysis of the SEP recording was not possible. The one patient who awakened despite bilaterally missing waveforms had a hearing deficit and used a hearing aid. Thus, in this study the addition

Table 2. Characteristics of somatosensory evoked potential (SEP) recordings

Median Value (Interquartile Range)

\section{Characteristic}

Temperature during SEP recording, ${ }^{\circ} \mathrm{C}$

Nerve conduction velocity, $\mathrm{m} / \mathrm{sec}$

Latency to N13 response, msec, RS stimulation

Latency to N13 response, msec, LS stimulation

Latency to cortical N20 response, msec, RS stimulation

Latency to cortical N20 response, msec, LS stimulation

CCT, msec, RS stimulation

CCT, msec, LS stimulation

Amplitude of $\mathrm{N} 20$ response, $\mu \mathrm{V}$, RS stimulation

Amplitude of $\mathrm{N} 20$ response, $\mu \mathrm{V}$, LS stimulation

\begin{tabular}{ccc}
\hline Hypothermia Group $(\mathrm{n}=30)$ & Normothermia Group $(\mathrm{n}=27)$ & $p$ Value \\
$33.0(32.7-33.4)$ & $37.5(36.6-37.8)$ & \\
$46.2(42.9-48.5)$ & $53.5(49.2-56.9)$ & $<.001$ \\
$18.1(16.8-19.0)$ & $15.0(14.0-16.0)$ & $<.001$ \\
$17.5(16.5-19.0)$ & $14.8(14.0-16.0)$ & $<.001$ \\
$25.0(24.0-26.8)$ & $21.0(20.0-22.0)$ & $<.001$ \\
$24.7(23.3-26.0)$ & $20.5(20.0-21.2)$ & $<.001$ \\
$7.2(6.5-7.8)$ & $6.0(5.5-6.5)$ & $<.001$ \\
$7.0(6.5-7.5)$ & $6.0(5.0-6.3)$ & NS \\
$1.3(1.0-1.7)$ & $1.4(1.2-1.9)$ & NS \\
$1.1(1.0-1.5)$ & $1.4(0.9-1.8)$ &
\end{tabular}

RS, right-sided; LS, left-sided; CCT, cervicocortical conduction time; NS, not significant. 
Table 3. Relation between outcome and the presence of N20 responses in somatosensory evoked potential (SEP) recordings

\begin{tabular}{|c|c|c|}
\hline \multirow[b]{2}{*}{ Variable } & \multicolumn{2}{|c|}{ No. of Patients } \\
\hline & $\begin{array}{l}\text { Hypothermia Group } \\
\qquad(\mathrm{n}=30)\end{array}$ & $\begin{array}{l}\text { Normothermia Group } \\
\qquad(\mathrm{n}=30)\end{array}$ \\
\hline No. of patients with analyzable SEP recordings & 30 & 27 \\
\hline Awakened, N20 responses present & 26 & 17 \\
\hline \multicolumn{3}{|l|}{ Outcome at $6 \mathrm{mos}$} \\
\hline CPC 1 & 20 & 9 \\
\hline CPC 2 & 3 & 4 \\
\hline CPC 3 & 2 & 3 \\
\hline CPC 4 & 0 & 0 \\
\hline CPC 5 & 1 & 1 \\
\hline Awakened, N20 responses absent & 0 & 0 \\
\hline Remained in coma, $\mathrm{N} 20$ responses present & 1 & 2 \\
\hline \multicolumn{3}{|l|}{ Outcome at 6 months } \\
\hline CPC 5 & 1 & 2 \\
\hline Remained in coma, $\mathrm{N} 20$ responses absent & 3 & 8 \\
\hline \multicolumn{3}{|l|}{ Outcome at 6 months } \\
\hline CPC 4 & 0 & 1 \\
\hline CPC 5 & 3 & 7 \\
\hline
\end{tabular}

CPC, cerebral performance category.

Table 4. Characteristics of brain stem auditory evoked potential (BAEP) recordings

\begin{tabular}{lccc}
\hline & \multicolumn{2}{c}{ Median (Interquartile Range) } & \\
\cline { 2 - 3 } \multicolumn{1}{c}{ Characteristic } & $\begin{array}{c}\text { Hypothermia Group } \\
(\mathrm{n}=30)\end{array}$ & $\begin{array}{c}\text { Normothermia Group } \\
(\mathrm{n}=30)\end{array}$ & $p$ Value \\
\hline Temperature during BAEP recording, ${ }^{\circ} \mathrm{C}$ & $33.0(32.7-33.4)$ & $37.5(37.2-37.8)$ & \\
Latency of wave I, msec, RS stimulation & $1.85(1.77-1.99)$ & $1.70(1.60-1.94)$ & .02 \\
Latency of wave I, msec, LS stimulation & $1.85(1.69-1.97)$ & $1.64(1.55-1.80)$ & .005 \\
Latency of wave III, msec, RS stimulation & $4.59(4.33-4.73)$ & $4.08(3.89-4.23)$ & $<.001$ \\
Latency of wave III, msec, LS stimulation & $4.54(4.29-4.91)$ & $4.06(3.84-4.13)$ & $<.001$ \\
Latency of wave V, msec, RS stimulation & $6.88(6.61-7.10)$ & $6.08(5.78-6.44)$ & $<.001$ \\
Latency of wave V, msec, LS stimulation & $6.90(6.71-7.19)$ & $5.88(5.64-6.51)$ & $<.001$ \\
Interpeak latency I-III, msec, RS stimulation & $2.70(2.53-2.87)$ & $2.32(2.09-2.43)$ & $<.001$ \\
Interpeak latency I-III, msec, LS stimulation & $2.73(2.54-3.04)$ & $2.33(2.19-2.48)$ & $<.001$ \\
Interpeak latency III-V, msec, RS stimulation & $2.28(2.14-2.48)$ & $1.99(1.85-2.14)$ & $<.001$ \\
Interpeak latency III-V, msec, LS stimulation & $2.35(2.07-2.50)$ & $1.94(1.75-2.19)$ & $<.001$ \\
Interpeak latency I-V, msec, RS stimulation & $5.01(4.76-5.25)$ & $4.33(4.08-4.57)$ & $<.001$ \\
Interpeak latency I-V, msec, LS stimulation & $5.13(4.86-5.35)$ & $4.22(3.89-4.45)$ & $<.001$ \\
\hline
\end{tabular}

RS, right-sided; LS, left-sided.

of BAEP recording to SEP recording did not increase sensitivity for identifying patients with poor prognosis, as compared with SEP recording alone.

\section{DISCUSSION}

Our results suggest the possibility that the prognostic ability of median nerve SEP recordings is preserved in cardiac arrest patients treated with hypothermia of $33^{\circ} \mathrm{C}$. The bilateral absence of early N20 cortical responses also was a reliable predictor of permanent coma after cardiac arrest in these patients. The high sensitivity is most likely attributed to the small number of patients.

Therapeutic hypothermia significantly reduces the nerve conduction velocities similar but decreased significantly at a temperature of $27.8^{\circ} \mathrm{C}$.

SEP and BAEP recordings are noninvasive, reproducible techniques that can be easily performed at the ICU bedside. Induced hypothermia requires muscle relaxation, which prevents the adjustment of SEP stimuli intensity on the basis of thumb twitch. The intensity of the stimuli has to be sufficient to evoke an ipsilateral supraclavicular response in patients with clinical muscle relaxation. Significant improvements in SEP have been reported within 24 hrs after ROSC (18), and allowing a period of at least 24 hrs after cardiac arrest is recommended for obtaining a reliable prognosis based on SEP. Short-latency SEPs and BAEPs are quite resistant to anesthetic agents. In the study of McPherson et al. (19), thiopental increased the latency of N20 and an intravenous bolus of fentanyl 25 $\mu \mathrm{g} / \mathrm{kg}$ increased the latency of N20 and decreased the amplitude of N20. In our study the dosage of fentanyl was only 2 $\mu \mathrm{g} / \mathrm{kg}$ intravenously every hour. For BAEP, fentanyl with doses up to $50 \mu \mathrm{g} / \mathrm{kg}$ intravenously had no significant effect on absolute or interpeak latencies of wave I-, III-, or V-evoked potentials (20).

Theoretically, abnormal BAEP would not be expected to be a sensitive marker of neuronal damage after global ischemia, since cortical neurons are more sensitive to hypoxic-ischemic damage than neurons in the brainstem. According to our results, BAEP recordings are not useful in identifying a poor prognosis for cardiac arrest patients either when used alone or when used in combination with shortlatency SEPs. Missing waveforms in BAEP can indicate a brainstem lesion, but this finding may also be attributed to hearing loss, which is a common confounding factor, especially in elderly patients.

There are some limitations to this study. Because of the strict inclusion and exclusion criteria, these patients represent only a minor proportion of all resuscitated cardiac arrest patients, and thus the results cannot be applied to all resuscitated patients treated with hypothermia. Still, this is the patient group in which studies have shown therapeutic hypothermia to be effective in ameliorating neurologic outcome after cardiac arrest $(1,2)$. The number of patients was relatively small and the proportion of patients whose outcome was favorable was exceptionally high. Although the target population was not identical with that of 


\section{he prognostic ability of median nerve short-latency} somatosensory evoked potentials does not seem to be affected by therapeutic hypothermia, and brainstem auditory evoked potentials had no additional value in outcome prediction.

the Hypothermia After Cardiac Arrest trial, there was no additional bias in patient selection. One limitation of this study is the relatively small proportion of patients not recovering consciousness. Also, the number of patients whose outcome was poor and who had detectable N20 SEP peaks was small. The power of this study is not sufficient enough to detect a possible correlation between outcome and N20 peak latencies or N20 amplitudes. In normothermic cardiac arrest survivors, hypoxic-ischemic brain damage with poor outcome has been shown to be associated with prolonged latencies and decreased amplitudes of SEP N20 peaks (21). Short-latency SEPs lack the capacity to identify patients whose outcomes will be favorable after cardiac arrest. Long-latency SEPs have been found useful even for this purpose by Austrian investigators (22). Madl et al. have previously shown that in normothermic cardiac arrest patients, a latency $>130 \mathrm{msec}$ to cortical evoked potential N70 peak or absent N70 peaks predict poor outcome with a specificity of $97 \%$ and sensitivity of $94 \%$. They also found that the N70 longlatency sensory evoked potential was more accurate in predicting individual outcome than was a panel of three experienced emergency physicians reviewing clinical data 24 hrs after cardiac arrest (23). Because of the slowing of neural transmission with hypothermia, the N70 cut-off values determined for normothermic patients cannot be applied to hypothermic patients.

To avoid falsely pessimistic prognosis, tests used in critical care are required to have high specificity for poor outcome, with less emphasis on sensitivity. Induced hypothermia requires muscle relaxation, sedation, and mechanical ventilation, which may complicate the clinical assessment. Reliable tests predicting unfavorable neurologic outcome for these patients are needed. In a previous study, we found that the use of therapeutic hypothermia reduces the prognostic value of both serum neuron-specific enolase and S-100B protein in predicting poor outcome after cardiac arrest (24). This small study suggests that the prognostic ability of median nerve short-latency SEPs may not be affected by therapeutic hypothermia. Recording of BAEPs did not provide additional benefit in outcome prediction. Larger trials are needed to confirm these findings.

\section{ACKNOWLEDGMENT}

We thank Prof. Seppo Sarna for reviewing the statistical analysis.

\section{REFERENCES}

1. The Hypothermia After Cardiac Arrest Study Group: Mild therapeutic hypothermia to improve the neurologic outcome after cardiac arrest. N Engl J Med 2002; 346:549-556

2. Bernard S, Gray T, Buist M, et al: Treatment of comatose survivors of out-of-hospital cardiac arrest with induced hypothermia. $N$ Engl J Med 2002; 346:557-563

3. Zandbergen E, de Haan R, Stoutenbeek C, et al: Systematic review of early prediction of poor outcome in anoxic-ischaemic coma. Lancet 1998; 352:1808-1812

4. Robinson LR, Micklesen PJ, Tirschwell DL, et al: Predictive value of somatosensory evoked potentials for awakening from coma. Crit Care Med 2003; 31:960-967

5. Markand ON, Warren C, Mallik GS, et al: Temperature-dependent hysteresis in somatosensory and auditory evoked potentials. Electroencephalogr Clin Neurophysiol 1990; 77:425-435

6. Coles JG, Taylor MJ, Pearce JM, et al: Cerebral monitoring of somatosensory evoked potentials during profoundly hypothermic circulatory arrest. Circulation 1984; 70:96-102

7. Zeitlhofer J, Steiner M, Bousek K, et al: The influence of temperature on somatosensoryevoked potentials during cardiopulmonary bypass. Eur Neurol 1990; 30:284-290

8. Porkkala T, Kaukinen S, Häkkinen V, et al: Effects of hypothermia and sternal retractors on median nerve somatosensory evoked potentials. Acta Anesthesiol Scand 1997; 41: 843-848

9. Markand ON, Warren C, Mallik GS, et al: Effects on hypothermia on short latency somatosensory evoked potentials in humans. Electroencephalogr Clin Neurophysiol 1990; 77:416-424
10. Guerit JM, Verhelst R, Rubay J, et al: The use of somatosensory evoked potentials to determine the optimal degree of hypothermia during circulatory arrest. J Card Surg 1994; 9:596-603

11. Stockard JJ, Sharbrough FW, Tinker JA: Effects of hypothermia on the human brainstem auditory response. Ann Neurol 1978; 3:368-370

12. Markand ON, Lee BI, Warren C, et al: Effects of hypothermia on brainstem auditory evoked potentials in humans. Ann Neurol 1987; 22:507-513

13. Cummins RO, Chamberlain DA, Abramson NS, et al: Recommended guidelines for uniform reporting of data from out-of-hospital cardiac arrest: the Utstein Style: a statement for health professionals from a task force of the American Heart Association, the European Resuscitation Council, the Heart and Stroke Foundation of Canada, and the Australian Resuscitation Council. Circulation 1991; 84:960-975

14. Jennet B, Bond M: Assessment of outcome after severe brain damage. Lancet 1975; 1:480-484

15. Brain Resuscitation Clinical Trial II Study Group: A randomized clinical study of a calcium-entry blocker (lidoflazine) in the treatment of comatose survivors of cardiac arrest. N Engl J Med 1991; 324:1225-1231

16. Benita M, Conde H: Effects of local cooling upon conduction and synaptic transmission. Brain Res 1972; 36:133-151

17. Buchtal F, Rosenfalck A: The influence of temperature on the sensory action potential and its conduction. Brain Res 1966; 3:54-55

18. Gendo A, Kramer L, Häfner M, et al: Timedependency of sensory evoked potentials in comatose cardiac arrest survivors. Intensive Care Med 2001; 27:1305-1311

19. McPherson RW, Sell B, Traystman RJ: Effects of thiopental, fentanyl, and etomidate on upper extremity somatosensory evoked potentials in humans. Anesthesiology 1986; 65: 584-589

20. Samra SK, Lilly DJ, Rush NL, et al: Fentanyl anesthesia and human brain-stem auditory evoked potentials. Anesthesiology 1984; 61: 261-265

21. Bauer E, Funk G-C, Gendo A, et al: Electrophysiological assessment of the afferent sensory pathway in cardiac arrest survivors. Eur J Clin Invest 2003; 33:283-287

22. Madl C, Grimm G, Kramer L, et al: Early prediction of individual outcome after cardiopulmonary resuscitation. Lancet 1993; 341:855-858

23. Madl C, Kramer L, Domanovits H, et al: Improved outcome prediction in unconscious cardiac arrest survivors with sensory evoked potentials compared with clinical assessment. Crit Care Med 2000; 28:721-726

24. Tiainen M, Roine RO, Pettilä V, et al: Serum neuron-specific enolase and S-100B protein in cardiac arrest patients treated with hypothermia. Stroke 2003; 34:2881-2886 